\title{
POLAR TRANSMITTER FOR WIRELESS COMMUNICATION SYSTEM
}

\author{
Chung-Chun Chen, Hung-Yang Ko, Yi-Chiuan Wang, Hen-Wai Tsao, Kai-Yuan Jheng, and An-Yeu (Andy) Wu
}

Graduate Institute of Electronics Engineering, National Taiwan University, Taipei 10617, Taiwan, ROC

\begin{abstract}
Polar modulation techniques offer the capability of multimode wireless system and the potential for the high efficiency Power Amplifier (PA). Any input baseband complex signal is decomposed into magnitude and phase signal, and goes through envelope modulator and phase modulator respectively. The modulated envelope and phase message signals are combined and amplified by switched-mode PA. In this paper, we will focus on the rectangular-to-polar converter, envelope modulator and phase modulator of polar transmitter for EDGE (2.5G) system. The analog part includes open-loop envelope modulator. The digital part includes rectangular-topolar converter and digital phase modulator. We employ the Coordinate Rotation Digital Computer (CORDIC) and Direct Digital Frequency Synthesizer (DDFS) techniques in this part. A prototype chip has been designed and fabricated in $U M C$ $0.18 \mathrm{um}$ CMOS process with 1 P6M technology.
\end{abstract}

\section{INTRODUCTION}

Polar modulation offers the capability of achieving high linearity and high efficiency simultaneously in a wireless transmitter. Improved efficiency is achieved by using a highly efficient and non-linear PA to work at its peak efficiency. Linear transmission is achieved by modulating the envelope of the signal through the voltage supply of the PA.

Polar transmission utilizes envelope and phase component to represent the digital symbols instead of the conventional $I / Q$ format [1]. The baseband signal $V(t)$ is split into the phase signal $\theta(t)$ and the envelope signal $A(t)$

$$
\begin{aligned}
V(t) & =x(t)+j \cdot y(t) . \\
A(t) & =\sqrt{x(t)^{2}+y(t)^{2}}, \\
\theta(t) & =\tan ^{-1}\left(\frac{y(t)}{x(t)}\right) .
\end{aligned}
$$

It is clear that from Eq. (2) we can have a phase-only signal through phase modulator and multiplied with its envelope at the PA to restore the original complex signal $V(t)$. This polar modulation process is like the Envelope Elimination and Restoration (EER) [2] architecture. The Eq.(2) is done by a limiter and an envelope detector. But the circuits will suffer from the non-linearity and distortion of the analog devices and would cause mismatch problem through the two paths. In this paper we proposed a DSP engine which includes rectangular-to-polar converter and digital Phase Modulator (PM). The design does not have the distortion problem caused by the analog components and the phase modulation process can be precisely controlled by the digital phase modulator. The baseband phase signal is modulated through digital phase modulator at the specific frequency range. The phase modulated signal is represented as $\mathrm{S}_{I F-P M}(t)$.

$$
S_{I F-P M}(t)=\cos \left(w_{c} t+\phi(t)\right) .
$$

The PA stage of amplitude modulator (AM) operates in principle as a multiplier in our design model. This gives the output signal in the specific frequency band as follows:

$$
\begin{aligned}
s_{I F}(t) & =A(t) \cdot s_{I F-P M}(t), \\
& =A(t) \cdot \operatorname{Re}\left\{e^{j \phi(t)} \cdot e^{j w_{c} t}\right\}, \\
& =x(t) \cos \left(w_{c} t\right)+j \cdot y(t) \sin \left(w_{c} t\right) .
\end{aligned}
$$

For convenience of the simulation model [2], the gain of the PA is set to one. Thus the Eq. (4) is equal to the signal of EDGE, which is up-converted at Intermediated Frequency (IF) band. The nonlinearity of PA and analysis of up-converter to Radio Frequency (RF) stage are beyond the scope of this paper.

\section{POLAR TRANSMITTER ARCHITECTURE}

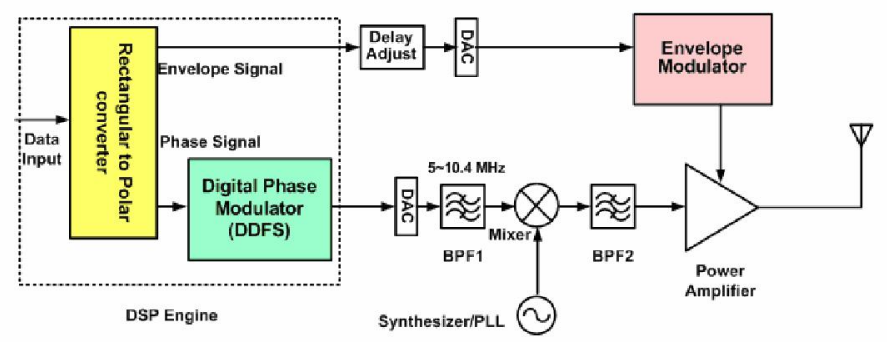

Fig. 1. Architecture of polar transmitter.

The architecture of the polar transmitter is shown in Fig. 1. The rectangular-to-polar converter extracts the phase and envelope information of a symbol in the digital domain. Then the envelope signal is modulated by envelope modulator and fed through the voltage supply of the PA. The phase information is modulated by digital phase modulator to create a constant envelope and phase modulated signal. The phase modulated precision and channel selection can be well controlled in the digital part first. In this paper we use the concept from [3] to realize the digital phase modulator design. The digital fine-tune frequencies are generated by the DDFS. The DDFS interpolates the carrier frequencies between the coarse frequencies generated by the integer-N PLL. The clock rate of the DDFS can be derived as in [3]. In our design, the clock rate of DDFS will be operated at $26 \mathrm{MHz}$. The digital fine tuning frequencies are generated by the DDFS and locating at $5 \mathrm{MHz} 10.4 \mathrm{MHz}$. Each interpolated frequency (channel) is stored in the fine-tune Frequency Control Word (FCW) table. 


\section{RECTANGULAR-TO-POLAR CONVERTER}

For a coordinate axis converter, we adopt the CORDIC algorithm in our design since the algorithm is very simple and low hardware cost. In order to further reduce the complexity, we also apply the technique in [4] to our rectangular-to-polar converter. For the first iteration we move the input vector into $l_{-t h}$ and $4_{-t h}$ quadrant with simply sign inversing and data exchanging. Second we replace $y_{i}$ by $y_{i} \cdot 2^{-i}$ as compared with conventional CORDIC algorithm. This modification can save once iteration and one barrier shifter in the rectangular-to-polar converter. This can save more area in our design. The iterations (for $i=2 \sim n$ ) are shown in Eq. (5).

$$
\begin{aligned}
x_{i+1} & =x_{i}+d_{i} \cdot 2^{-2(i-2)} \cdot y_{i}, \\
y_{i+1} & =2 \cdot\left[y_{i}-d_{i} x_{i}\right] \\
z_{i+1} & =z_{i}+d_{i} \cdot p_{i} \cdot \\
p_{i} & =\frac{1}{\pi} \cdot \tan ^{-1}\left(2^{-(i-2)}\right), \\
K & =\frac{1}{\sqrt{\sum_{i} 1+2^{-2(i-2)}}} .
\end{aligned}
$$

The desired phase is $z_{i+1}$ and the desired envelope value is $x_{i+1}$ multiplied by a constant scaling factor $K$. Due to the iterative feature of CORDIC algorithm, the clock rate of this module is $\mathrm{n}^{*} \mathrm{f}_{\mathrm{clk}}$, and $\mathrm{n}$ is iteration number. It is hard for the module to operate at such high clock rate. A compromise is to use unfolded technique and the architecture is shown in Fig. 2.

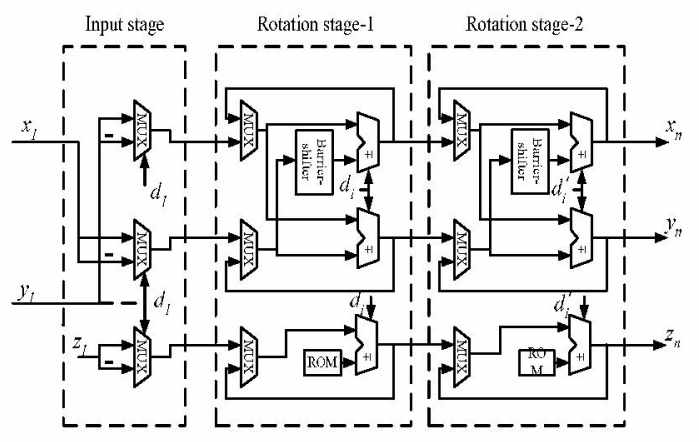

Fig. 2. Architecture of rectangular-to-polar converter.

\section{DIGITAL PHASE MODULATOR}

The DDFS architecture is shown in Fig. 3. The DDFS has three basic blocks: FCW table, phase accumulator and phase-toamplitude converter. The FCW table stores the desired fine-tune frequency control words and can be derived from Eq. (6).

$$
f_{c}=\frac{F C W \cdot f_{\text {clk }}}{2^{L}}, \quad \forall \quad F C W<2^{L-1}
$$

In our design we focus on the phase-to-amplitude converter design which is based on Least Squared (LS) algorithm [5] and Merged-Multiply Accumulator (MAC) technique [6]. The input phase is truncated by 3 -bit according to the $\pi / 4$ symmetry and the amplitude of the sine function can be express by the polynomial.
The approximated polynomial is generated according to the LS algorithm. In this paper we compare the Spurious Free Dynamic Range (SFDR) performance with the other approximation algorithm such as Taylor and Chebyshev [9]. The comparison method is set the input phase from 0 to $\pi / 2$. The phase wordlength is 15-bit and amplitude output is 15-bit. From the simulation result in Fig. 4, we can see that the LS-based polynomial can achieve better performance than other approximation algorithm with less polynomial order. Less order of polynomial means that low hardware complexity can be achieved.

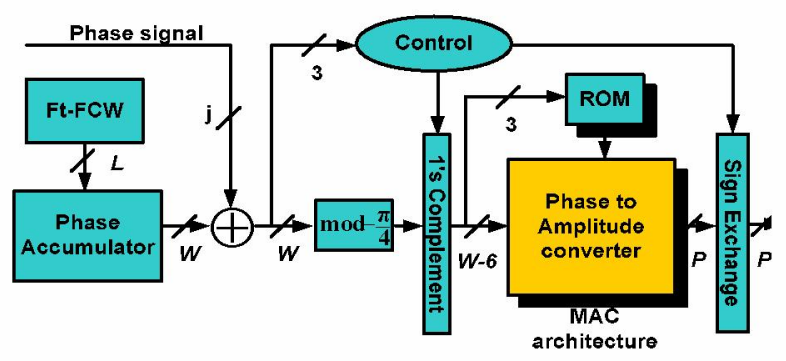

Fig. 3. Architecture of DDFS

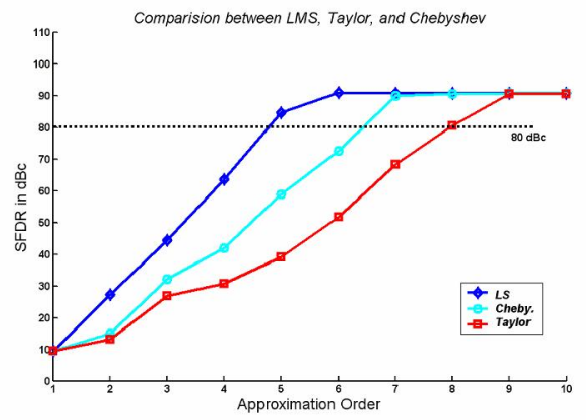

Fig. 4. SFDR comparison between LS, Taylor and Chebyshev.

In order to reduce the polynomial order we further divide the approximated region into eight segments. In each segment, the approximated polynomial $p(X)$ can be represented as in Eq. (7).

$$
\begin{aligned}
& p(X) \\
& =c_{2} \cdot X^{2}+c_{1} \cdot X+c_{0} \\
& =\sum_{i=0}^{n_{1}-1} R_{i} \cdot 2^{i}+\left[c_{1}\right]_{n_{2}} \cdot[X]_{n_{3}}+\sum_{k=0}^{n_{4}-1} C_{k} \cdot 2^{k} \\
& =M A C\left(\left[\text { rom }{ }_{-}\right]_{n_{1}}+\sum_{j=0}^{n_{2} / 2} Q_{j} \cdot[X]_{n_{3}} \cdot 4^{j}+[\text { rom_ } 2]_{n_{4}}\right) . \\
& Q_{j}=-2 c_{1,2 j+1}+c_{1,2 j}+c_{1,2 j-1}, \\
& c_{1, j}=0,1 \quad \text { and } c_{1,-1}=0, \\
& \quad[]_{n}: \text { denote the truncation with } n-\text { bit. }
\end{aligned}
$$

Where $\mathrm{c}_{\mathrm{i}}$ represents the coefficient, and $X$ is the phase offset of each divided region. In Eq. (7) we store the first term and third term in the look-up table. The operations in Eq. (7-8) now become one booth multiplication and two constant additions. These can be merged into a modified-MAC (Fig. 5). X is inputted to the booth decoder circuit and the partial product term is generated in each 
row of MAC. The partial product terms are summed through Carry-Save-Adder (CSA) tree. As compared with the direct implementation of $2^{\text {th }}$ order polynomial, the CSA tree can prevent the carry ripple problem in the early stages, and the carry ripple only occurs at the final stage. Due to the EDGE spectral requirement we target the desired SFDR over $80 \mathrm{dBc}$. From Matlab simulation, we set the truncated accumulated phase wordlength to $W=15$ bits and amplitude word-length to $P=14$ bits. These hardware parameters can achieve SFDR $=86 \mathrm{dBc}$. The wordlength of the phase of the EDGE signal will also introduce phase noise and spurs in the output spectrum and will be discussed in section 6. The proposed DDFS circuit is simulated by the NANOSIM tool and compares with state of the art in Table 1. The proposed DDFS can achieve high SFDR performance. The power efficiency is also superior to the other designs.

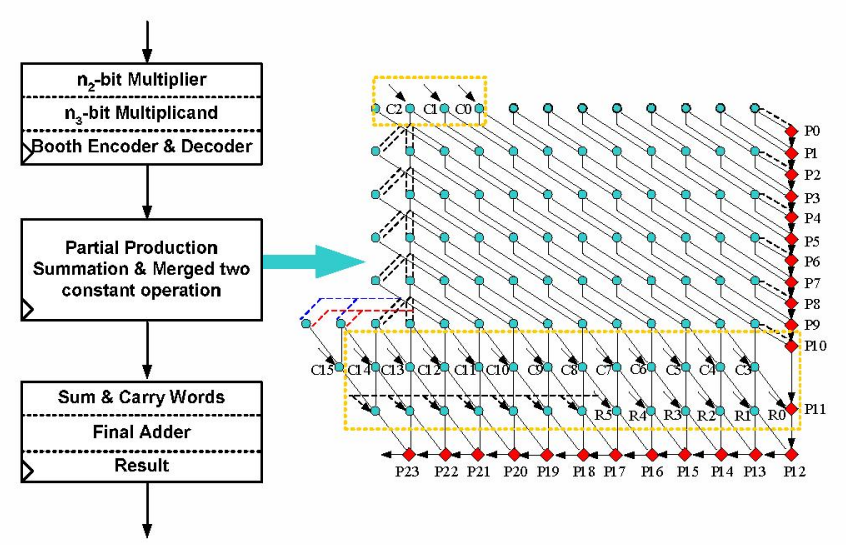

Fig. 5. Architecture of Modified-MAC.

Table 1. Comparison with the existing DDFS designs.

\begin{tabular}{|c|c|c|c|c|}
\hline DDFS & $\begin{array}{c}\text { CMOS } \\
\text { tech. }\end{array}$ & SFDR & Latency & $\begin{array}{c}\text { Power } \\
\text { efficiency } \\
\text { (mW/MHz) }\end{array}$ \\
\hline Ours & 0.18 & 86 & 5 & 0.15 \\
\hline Ref [7] & 0.18 & 84 & - & 0.22 \\
\hline Ref [8] & 0.25 & 90.3 & 13 & 0.66 \\
\hline $\begin{array}{c}\text { Ref [9] } \\
\text { (Taylor) }\end{array}$ & 0.35 & 82.5 & 9 & 0.26 \\
\hline $\begin{array}{c}\text { Ref [9] } \\
\text { (Chebyshev) }\end{array}$ & 0.35 & 73 & 7 & 0.35 \\
\hline Ref [10] & 0.35 & 80 & 2 & 0.44 \\
\hline
\end{tabular}

\section{ENVELOPE MODULATOR}

Because open-loop architecture can provide more bandwidth and less cost than close-loop architecture, we use an open loop approach to design the architecture of envelope modulator (Fig. 6). The pre-distorter is needed in the open loop architecture. Besides, the bandwidth and consideration of cost, the circuit integration is another big issue. The inductance of the switched-mode power supply is too large to set on chip. The integration problem may cause many design problems and cost issues. Another design issues we faced is that the speed of the switching of the power transistor. Based on above investigation, we decided to adopt the power supply named linear regulator shown in Fig. 7[11][12] at the cost of larger power dissipation. The linear regulator could provide faster switching rate and no inductance is needed. Before the linear regulator, a DAC is needed to convert the digital signal to analog signal.

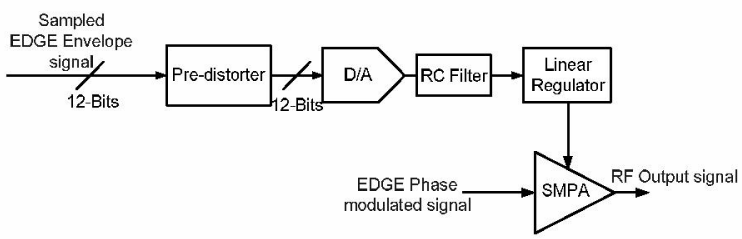

Fig. 6. Open loop architecture of envelope modulator

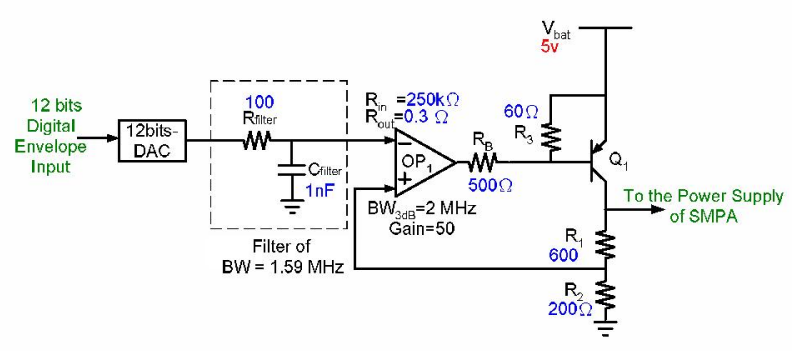

Fig. 7. Architecture of linear regulator with RC filter and DAC

We selected BCP69 [13], [14] as the power transistor called Q1. BCP69 can supply with high current up to $1 \mathrm{~A}$ and be linear voltage regulator in application. According to data sheet of Analog Device, we selected AD8036 as the comparator called OP1 in the preliminary design. AD8036 is an operation amplifier with low distortion, wide bandwidth voltage feedback clamp.

\section{SIMULATION RESULT}

For Mobile Station (MS), the requirements of EVM-rms and EVM-peak are below $9 \%$ and 30\%. For Base-Tranceiver Station (BTS) EVM-rms and EVM-peak are below 7\% and 22\%. The SFDR performance of the digital frequency synthesizer is suitable for the up-link and down-link spectral requirement. The phase signal word-length also contributes spurs and phase noise and affects the EVM and the signal spectrum. In this paper we simulate the finite word-length $(J)$ effect of the phase signal with the EVM measurement and spectral mask requirement. The performance summary is in Table 2 .

Table 2. Simulation result and EVM measurement.

\begin{tabular}{|c|c|c|c|}
\hline J-bits & EVM-rms & EVM-peak & Spectral requirement \\
\hline 9-bits & $0.028 \%$ & $0.094 \%$ & No (Spurs at -66dBc) \\
\hline 10-bits & $0.014 \%$ & $0.046 \%$ & No (Spurs at -74dBc) \\
\hline 11-bits & $0.007 \%$ & $0.018 \%$ & No (Spurs at -79dBc) \\
\hline 12-bits & $0.003 \%$ & $0.011 \%$ & Yes (Spurs at -81dBc) \\
\hline
\end{tabular}

From the Table 2, we can see that the errors produced by the phase quantization are very small for the word-length higher than 9-bits. But the spectrum of the $S_{I F-P M}(t)$ signal is not exactly below the spectral mask. Especially for BTS-mask, the requirement of the mask is more stringent than MS-mask. It is conservative to choose $J=12$-bit in our design. The simulation is based on the architecture of the polar transmitter in the ADS environment. The 
simulation results show the performance of the polar transmitter system for EDGE in the condition of PA nonlinear model. Because of the nonlinearity, we adopted the pre-distortion method to linearize the PA. Fig. 8 shows the simulation block of nonlinear model with predistorter. The final signal spectrum is shown in Fig. 9 and can meet the spectral requirement for BTS-mask and MSmask.

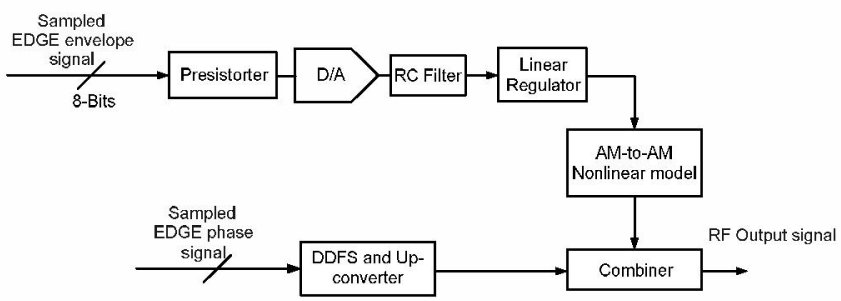

Fig. 8. Simulation block of polar transmitter with nonlinear model and with predistorter

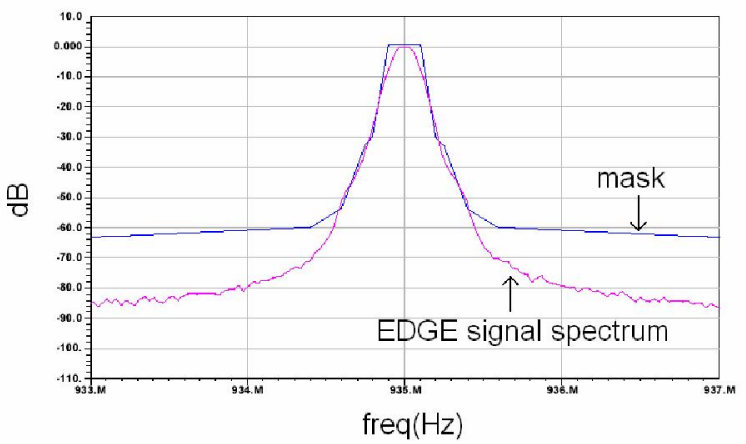

Fig. 9. The final spectrum of the EDGE signal

\section{IMPLEMENTATION RESULT}

The proposed DSP engine is implemented in UMC $0.18 \mathrm{um}$ CMOS process with IPOM technology. The layout is shown in Fig. 10. The summary of the circuit is list in Table 3.

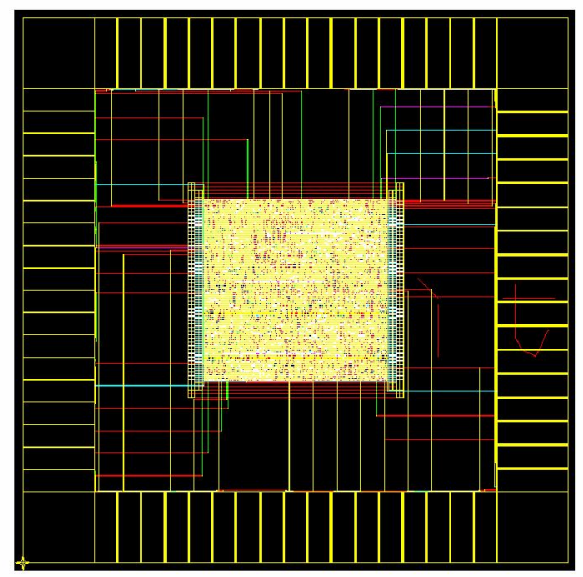

Fig. 10. layout of the proposed DSP engine.

Table 3. Implement summary of the DSP engine.

\begin{tabular}{|c|c|}
\hline Technology & UMC 0.18 um 1P6M CMOS \\
\hline Voltage & $1.8 \mathrm{~V}$ \\
\hline Core layout area & $0.51 \times 0.51 \mathrm{~mm}^{2}$ \\
\hline Chip layout area & $1.114 \times 1.114 \mathrm{~mm}^{2}$ \\
\hline System clock Frequency & $26 \mathrm{MHz}$ \\
\hline Power consumption $026 \mathrm{MHz}$ & $3.92 \mathrm{~mW}$ \\
\hline
\end{tabular}

\section{CONCLUSION}

In this paper, we propose a DSP engine for the polar transmitter. The engine is realized by the CORDIC and DDFS techniques. In the digital phase modulator we adopt the LS algorithm. We also apply MAC technique in our DDFS architecture to reduce the hardware complexity and decrease the carry ripple problem of the direct polynomial implementation. We adopt the architecture of linear regulator in the envelope modulator design. The open loop architecture is suitable for mobile handset and wideband modulation scheme. The chip implementation with $U M C 0.18 \mathrm{um}$ CMOS process with $I P 6 M$ technology is also presented in this paper.

\section{ACKNOWLEDGEMENT}

This work was supported by MediaTek Inc., under NTU-MTK wireless research project.

\section{REFERENCES}

[1] P. Nagle, P. Burton, E. Heaney, and F. McGrath, "A wide-band linear amplitude modulator for polar transmitters based on the concept of interleaving delta modulation," IEEE Journal of Solid-State Circuits, vol. 37, pp. 1748-1756, Dec. 2002.

[2] D. Rudolph, "Out-of-band emissions of digital transmissions using Kahn EER technique," IEEE Trans. Microwave Theory and Techniques, vol. 50, pp. 1979-1983. Aug. 2002.

[3] J. Vankka, "Digital frequency synthesizer/modulator for continuousphase modulations with slow frequency hopping," IEEE Trans. Vehicular Technology, vol. 46, pp. 933-940, Nov. 1997.

[4] A. Chen and S. Yang, "Reduced complexity CORDIC demodulator implementation for D-AMPS and digital IF-sampled receiver," in Proc. Globecom '98, vol.3, Nov. 1998, pp.1491-1496.

[5] M. Flickner, J. Hafner, E.J. Rodriguez, and J.L.C. Sanz, "Fast leastsquares curve fitting using quasi-orthogonal splines," in Proc of IEEE Int. Image Processing, vol. 1, pp. 686-690, Nov. 1994.

[6] F. Elguibaly, "A fast parallel multiplier-accumulator using the modified Booth algorithm," IEEE Trans. Circuits and Systems, vol. 47 , pp. 902-908, Sept. 2000.

[7] J.M.P. Langlois and D. Al-Khalili, "Low power direct digital frequency synthesizers in $0.18 / \mathrm{spl} \mathrm{mu} / \mathrm{m}$ CMOS," in Proc of IEEE CICC, pp. 21-24, Sept. 2003.

[8] A. Torosyan, Dengrwei $\mathrm{Fu}$ and A. N. Willson, "A $300 \mathrm{MHz}$ quadrature direct digital synthesizer/mixer in $0.25 \mu \mathrm{m} \mathrm{CMOS,"} \mathrm{in}$ IEEE Solid-State Circuits Conference, vol. 1, pp. $132-133,2002$.

[9] K. I. Palomaki and Jarkko Niittylahti, "Phase-to-Amplitude Mapping in Direct Digital Frequency Synthesizers Using Series Approximation," EURASIP Journal on Applied Signal Processing, 2001.

[10]D. De Caro, E. Napoli and A. G. M. Strollo, "Direct digital frequency synthesizers using high-order polynomial approximation," in Proc of IEEE Solid-State Circuits Conference, vol. 1, pp. 134-135, 2002.

[11]W. Sander, Saturation prevention and amplifier distortion reduction, US patent 6,528,975, to Tropian Inc., Patent and Trademark Office, Washington, D.C, 2003.

[12] Wendell B. Sander, Stephan V. Schell, Brian L. Sander, Polar modulator for multi-mode cell phones, Tropian Inc., 20813 Stevens Creek Blvd. Cupertino, CA95014 USA.

[13] http://www.semiconductors.philips.com/acrobat/datasheets/BCP69 5 . pdf

[14] http:/www.semiconductors.philips.com/models/spicespar/data/BCP6 9.html 